Pacific Journal of Mathematics

DE RAM THEOREM WITH CUBICAL FORMS 


\title{
DE RHAM THEOREM WITH CUBICAL FORMS
}

\author{
Bohumil Cenkl and Richard Porter
}

\begin{abstract}
With a simplicial complex $X$ there is associated a commutative differential graded algebra of polynomial differential forms $T(X)$ together with a filtration $T^{*, q}(X) \subset T^{*, q+1}(X)$ in each degree ${ }^{*} . T^{*, q}(X)$ is a differential graded module over the subring of the rationals $\mathrm{Z}\left[\frac{1}{2}, \ldots, \frac{1}{q}\right]$. The deRham theorem for such a complex $T(X)$ is proved. We have demonstrated elsewhere that the refined deRham complex $T(X)$ makes it possible to substantially refine most of the results of the rational homotopy theory. In particular we defined the homotopy category of $C-N$ spaces which is equivalent to an algebraic homotopy category of $(N-1)$ connected free commutative differential graded algebras over the integers, satisfying a simple algebraic condition on cohomology.
\end{abstract}

1. Introduction. As a refinement of the deRham complex of rational differential forms, $A^{*}(X ; Q)$ on a simplicial complex, Cartan [1] and Miller [11] defined a filtration $A^{*, q}(X ; Z)$ of $A^{*}(X ; Q)$ such that the cohomology $H^{p}\left(A^{*, q}(X ; Z)\right)$ is isomorphic to the singular cohomology $H^{p}(X ; Z)$ for $p \leq q$. In our study, [2], [3], of the relationship between the fundamental group of $X$ and the filtered algebra $A^{* * *}(X ; Z)$, the best results are obtained under the assumption that $H_{1}(X ; Z)$ is both finitely generated and free. An effort to eliminate the freeness assumption lead us to the construction of a new commutative filtered algebra of forms, $T^{*, *}(X) . T^{*, *}(X)$ is an analogue of the filtered Cartan-Miller forms obtained by replacing simplices with cubes. Since each simplicial complex has a canonical subdivision into cubes, $T^{*, *}(X)$ can be viewed as a functor defined on simplicial complexes. For fixed $q, T^{*, q}(X)$ is a complex of $Q_{q}$ modules where $Q_{q}$ denotes the smallest subring of the rationals containing $1 / p$ for each prime $p, p \leq q, Q_{0}=Q_{1}=Z$. The usual wedge product of forms induces a map $T^{*, q_{1}}(X) \otimes T^{*, q_{2}}(X) \rightarrow$ $T^{*, q_{1}+q_{2}}(X)$. The main result of this paper is that integration of forms over cubes induces an isomorphism $I: H^{p}\left(T^{*, q}(X)\right) \rightarrow H^{p}\left(X ; Q_{q}\right)$ for $q \geq 1$ and for all $p . I$ is multiplicative in the sense that the diagram

$$
\begin{array}{ccc}
H^{p_{1}}\left(T^{*, q_{1}}(X)\right) \otimes H^{p_{2}}\left(T^{*, q_{2}}(X)\right) & \stackrel{\wedge}{ } & H^{p_{1}+p_{2}}\left(T^{*, q_{1}+q_{2}}(X)\right) \\
I \otimes I \downarrow & & I \\
H^{p_{1}}\left(X ; Q_{q_{1}}\right) \otimes H^{p_{2}}\left(X ; Q_{q_{2}}\right) & \stackrel{\cup}{ } & H^{p_{1}+p_{2}}\left(X ; Q_{q_{1}+q_{2}}\right)
\end{array}
$$

commutes. 
In [2]-[6] the $T$-forms are used to set up a correspondence between spaces and commutative differential graded algebras over the integers. For nonsimply connected spaces the algebraic model is equivalent to the Lazard completion of the fundamental group. For certain categories of C.W. complexes of finite type the $T$-forms induce an equivalence between the ordinary homotopy category of such C.W. complexes and a homotopy category of free commutative differential graded algebras over the integers. The homotopy category of tame spaces [7] of finite type is equivalent to a homotopy category of free commutative differential graded algebras over the integers, also of finite type.

The key technical difference between the $T$-forms and the filtered version of Cartan-Miller forms is that integration of $T$-forms induces an isomorphism in all dimensions, while integration of the filtered CartanMiller forms $A^{*, q}$ induces an isomorphism only in dimensions $\leq q$. As a consequence the algebraic models of simply connected spaces constructed in [4]-[6] contain more information about torsion in homotopy than the analogous models constructed using filtered Cartan-Miller forms. The algebraic model of the fundamental group based on $T$-forms [2]-[4] contains more information about $\pi$ than is contained in the corresponding model based on filtered Cartan-Miller forms unless the first homology group is torsion free, in which case the two models are the same (see [3]).

Lambe and Priddy [10] have used the filtered version of Cartan-Miller forms in the computation of $H^{*}\left(G ; Q_{q}\right)$ for certain finitely generated torsion free nilpotent groups $G$ and certain $q$.

More precise statements of applications of the $T$-forms to homotopy theory and some examples are given in $\$ 2$. Section 3 contains proofs of the properties of $T$-forms on a cube used in $\$ 4$ to prove the main result.

2. Applications and examples. In [2], [3], [5] the $T$-forms are used to associate a model, $M_{1}(X)$, to each connected space $X$ with $H_{1}(X ; Z)$ finitely generated. $M_{1}(X)$ is a free commutative differential graded algebra over the integers generated by elements in dimensions 1 and 2, and is called a 1-model for $X . M_{1}(X)$ in turn determines a space denoted by $\left|M_{1}(X)\right| . M_{1}(X)$ is an analogue, based on $T$-forms, of Sullivan's 1-minimal model [14] and is a stronger invariant than the 1-minimal model of $X$.

Invariants of $M_{1}(X)$ are related to the fundamental group of $X$. Given a 1-model $M_{1}$ set $L M_{1}$ equal to the first homology group of the chain complex $\operatorname{Hom}\left(Q M_{1}, Z\right)$, where $Q M_{1}$ denotes the indecomposables of $M_{1}$. As in [14] the quadratic part of the differential on $M_{1}$ induces a Lie algebra structure on $L M_{1}$. Denote by gr $L M_{1}=\bigoplus_{k \geq 1} \mathrm{gr}_{k} L M_{1}$ the associated graded Lie algebra. 
Given a group $G, \operatorname{gr}(G)=\bigoplus_{k \geq 1} \operatorname{gr}_{k}(G)$ denotes the graded Lie algebra of successive quotients of the lower central series subgroups of $G$ and $\operatorname{Laz}(G)$ denotes the Lazard completion of $G$. Roughly, a Lazard group is a group with just enough divisibility and completeness for the inverse of the Campbell-Hausdorff formula to be defined and give a Lie algebra structure on the group.

TheOREM 2.1. ([2], [3], [5]). Let $X$ be a connected space with $H_{1}(X ; Z)$ finitely generated. Then

(i) $\left|M_{1}(X)\right|$ is an Eilenberg-MacLane space of type $K\left(\operatorname{Laz}\left(\pi_{1}(X), 1\right)\right)$.

(ii) $\bigoplus_{k \geq 1}\left[\operatorname{gr}_{k} L M_{1}(X) \otimes Q_{k}\right]$ and $\bigoplus_{k \geq 1}\left[\operatorname{gr}_{k}\left(\pi_{1}(X)\right) \otimes Q_{k}\right]$ are isomorphic graded Lie algebras,

EXAMPLE 2.2. Let $G$ be the group of upper triangular matrices with $a$, $b$ integers and $c$ an element of $Z / 5 Z$.

$$
\left(\begin{array}{lll}
1 & a & c \\
0 & 1 & b \\
0 & 0 & 1
\end{array}\right)
$$

$G$ has presentation $\left\{g_{1}, g_{2}, g_{21}:\left(g_{21}, g_{1}\right),\left(g_{21}, g_{2}\right), g_{21}^{5},\left(g_{2}, g_{1}\right) g_{21}^{-1}\right\}$. Denote by $X$ the 2-dimensional C.W. complex corresponding to this presentation of $G$. Set $M_{1}(X)$ equal to the free commutative differential graded algebra over the integers with generators $w_{1}, w_{2}, w_{21}$ in dimension 1; $\gamma_{21}$ in dimension 2; and differential determined by setting $d w_{1}=d w_{2}=$ $0, d w_{21}=-w_{2} w_{1}+5 \gamma_{21}$. Then $M_{1}(X)$ is a 1 -model for $X . L M_{1}(X)$ is $Z \oplus Z \oplus Z / 5 Z$ with generators $\bar{w}_{1}, \bar{w}_{2}, \bar{w}_{21}$, respectively, and with Lie bracket given by $\left[\bar{w}_{2}, \bar{w}_{1}\right]=\bar{w}_{21},\left[\bar{w}_{21}, \bar{w}_{1}\right]=\left[\bar{w}_{21}, \bar{w}_{2}\right]=0$.

In this case, $G$ is a Lazard group so it follows from Theorem 2.1(i) that $\left|M_{1}(X)\right|$ is a $K(G, 1)$. Note that $G$ is isomorphic to the group obtained by applying the Campbell-Hausdorff formula to $L M_{1}(X)$. We conjecture, in general, that the Lazard completion of $\pi_{1}(X)$ is isomorphic to the group obtained by applying the Campbell-Hausdorff formula to the Lazard completion of $L M_{1}(X)$ as a Lie algebra.

In [4]-[6] the $T$-forms are used to associate a model $M(X)$ to each $N-1$ connected free commutative differential graded algebra over the integers called a model for $X . M(X)$ in turn determines a space $|M(X)|$. The construction of $M(X)$ is motivated by Sullivan's construction of minimal models [14] and is a stronger invariant than Sullivan's minimal model, as $M(X)$ contains information about torsion in the homotopy groups of $X$ and the Sullivan minimal model for $M(X) \otimes Q$ is the Sullivan minimal model for $X$. 
Given a free commutative differential graded algebra $M$ of finite type over the integers, set $L M$ equal to the homology groups of the chain complex $\operatorname{Hom}(Q M, Z)$. The quadratic part of the differential on $M$ induces a bracket [ , ]: $L_{p} M \otimes L_{q} M \rightarrow L_{p+q-1} M$ satisfying the usual identities for the Whitehead product in homotopy

(a)

$$
[x, y]=(-1)^{|x||y|}[y, x] \text {, }
$$

$$
(-1)^{|x| z \mid}[[x, y], z]+(-1)^{|x||y|}[[y, z], x]+(-1)^{|y| z \mid}[[z, x], y]=0 .
$$

In [7] Dwyer defines tame spaces and proves that the homotopy category of tame $(N-1)$ connected spaces, $N \geq 3$, is equivalent to an algebraic homotopy category of tame $N-1$ reduced differential graded Lie algebras over $Z$.

An $N-1$ connected space, $N \geq 3, X$ is called tame if $\pi_{N+k}(X)$ is an $S_{k}$-module for $k \geq 0$, where $S_{k}$ denotes the smallest subring of the rationals containing $1 / p$ for each prime $p$ with $2 p-3 \leq k$. We say $X$ is of finite type if each $\pi_{N+k}(X)$ is a finitely generated $S_{k}$-module. Associated to each $N-1$ connected space $X, N \geq 3$, there is a tame space $X_{T}$ and a map $f: X \rightarrow X_{T}$ inducing isomorphisms $f_{*}: \pi_{N+k}(X) \otimes S_{k} \rightarrow$ $\pi_{N+k}\left(X_{T}\right) . X_{T}$ is called the tame localization of $X$.

THEOREM 2.3 ([4], [5]). (i) The homotopy category of tame $N-1$ connected spaces, $N \geq 3$, of finite type is equivalent to an algebraic homotopy category of $N-1$ connected free commutative differential graded algebras of finite type over the integers.

(ii) Let $X$ be an $N-1$ connected space, $N \geq 3$, whose integer homotopy is of finite type and let $M(X)$ be a model for $X$. Then

(a) $\bigoplus_{k \geq 0}\left[L_{N+k} M(X) \otimes S_{k}\right]$ is isomorphic to $\bigoplus_{k \geq 0}\left[\pi_{N+k}(X) \otimes S_{k}\right]$ by an isomorphism which sends the bracket in $L M(X)$ to the Whitehead product in $\pi(X)$.

(b) $|M(X)|$ is the tame localization of $X$.

The $T$-forms induce an equivalence between ordinary homotopy theory of certain C.W. complexes of finite type and commutative differential algebras.

We say a graded group $H^{*}$ satisfies the $C-N$ condition if multiplication by $p$ defines an automorphism on $H^{N+k}$ for all pairs of positive integers $(p, k)$ with $2 p-3 \leq k+1$. 
A space $X$ is called a $C-N$ space if $X$ is $N-1$ connected and is homotopy equivalent to C.W. complex of finite type whose cohomology groups $H^{*}(X ; Z)$ satisfy the $C-N$ condition.

THEOREM 2.4 ([6]). For each integer $N \geq 3$ the homotopy category of $C-N$ spaces is equivalent to an algebraic homotopy category of $N-1$ connected free commutative differential graded algebras of finite type over the integers whose cohomology groups satisfy the $C-N$ condition.

EXAMPLE 2.5. Fix a positive integer $N$ which is not divisible by 2 and not divisible by 3 . Consider the problem of identifying all homotopy types of simply connected C.W. complexes whose integer cohomology ring is $H^{*}$, where

$$
H^{*}= \begin{cases}Z, & *=0,3, \\ Z / N Z, & *=4,8, \\ 0 & \text { otherwise }\end{cases}
$$

and the cup product $H^{4} \otimes H^{4} \rightarrow H^{8}$ is the zero map.

Given integers $\alpha_{1}, \alpha_{2}$ set $M\left(\alpha_{1}, \alpha_{2}\right)$ equal to the free commutative differential graded algebra over the integers with generators and differentials in dimension $\leq 8$ given below where the arrow points from a generator to its differential, and the number to the left of a generator indicates the dimension of the generator.

$$
\begin{aligned}
& u_{1} \rightarrow 0 \\
& u_{2} \rightarrow 0 \\
& u_{3} \rightarrow 0
\end{aligned}
$$

\begin{aligned} \hline \hline$(3) & v_{1} \rightarrow N u_{2} \\ (6) & v_{2} \rightarrow u_{1} u_{2} \\ (7) & v_{3} \rightarrow N u_{3} \\ (7) & v_{4} \rightarrow u_{2}^{2} \\ (10) & v_{5} \rightarrow u_{1} u_{3}\end{aligned}$

$$
\begin{aligned}
& w_{1} \rightarrow N v_{2}-v_{1} u_{1} \\
& w_{2} \rightarrow N v_{4}-v_{1} u_{2} \\
& w_{3} \rightarrow v_{2} u_{1} \\
& w_{4} \rightarrow N v_{5}-v_{3} u_{4}
\end{aligned}
$$

$$
\begin{aligned}
& x_{1} \rightarrow N w_{3}-w_{1} u_{1}-\alpha_{1} u_{3} \\
& x_{2} \rightarrow v_{2} v_{1}-w_{1} u_{2} \\
& y_{1} \rightarrow N x_{2}-w_{1} v_{1}-\alpha_{2} u_{3}
\end{aligned}
$$


Add additional generators as necessary so the cohomology vanishes in dimensions $\geq 9$. The construction of $M\left(\alpha_{1}, \alpha_{2}\right)$ is motivated by [9]. The double lines above separate generators of different resolution degree. Adapting the resolution-perturbation approach in [9], [12] to the present situation it follows from Theorem 2.4 that the set of homotopy types of simply connected C.W. complexes with cohomology rings $H^{*}$ are in a 1-1 correspondence with the set of algebraic homotopy types represented by the algebras $M\left(\alpha_{1}, \alpha_{2}\right)$. Here two free differential algebras are homotopy equivalent if there is a map between them inducing an isomorphism on cohomology.

Set $G$ equal to the group of lower triangular matrices with $a$ and $b$ units in $Z / N Z$ and $c$ an arbitrary element $\left(\begin{array}{ll}a & 0 \\ c & b\end{array}\right)$, in $Z / N Z$. Then $M\left(\alpha_{1}, \alpha_{2}\right)$, is homotopy equivalent to $M\left(\beta_{1}, \beta_{2}\right)$ if and only if there is an element $\left(\begin{array}{ll}a & 0 \\ c & b\end{array}\right)$ in $G$ with

$$
\left(\begin{array}{ll}
a & 0 \\
c & b
\end{array}\right)\left(\begin{array}{l}
\bar{\alpha}_{1} \\
\bar{\alpha}_{2}
\end{array}\right)=\left(\begin{array}{l}
\bar{\beta}_{1} \\
\bar{\beta}_{2}
\end{array}\right)
$$

where $\bar{\alpha}_{1}, \bar{\alpha}_{2}, \bar{\beta}_{1}, \bar{\beta}_{2}$ denote the $\bmod N$ reductions of $\alpha_{1}, \alpha_{2}, \beta_{1}, \beta_{2}$ respectively.

Thus the set of homotopy types of simply connected C.W. complexes of finite type with cohomology ring $H^{*}$ is in a $1-1$ correspondence with the set of orbits of $G$ acting on $Z / N Z \oplus Z / N Z$. This illustrates the general result that if the ring $H^{*}$ is $N-1$ connected, $N \geq 3$, finite dimensional and satisfies the $C-N$ condition, then the set of homotopy types of simply connected C.W. complexes of finite type with cohomology ring isomorphic to $H^{*}$ can be identified with the set of orbits of a unipotent group acting on a lattice in a finite-dimensional algebraic variety.

A cell complex corresponding to the algebra $M\left(\alpha_{1}, \alpha_{2}\right)$ is constructed as follows. Set $X_{1}$ equal to the wedge of spheres $S_{a}^{3} \vee S_{b}^{3} \vee S_{c}^{7}$ and let $\iota_{a}$, $\iota_{b}, \iota_{c}$ denote elements in $\pi\left(X_{1}\right)$ corresponding to the inclusions of the respective spheres. To $X_{1}$ attach a 4-cell by an attaching map representing the element $N \iota_{b}$ in $\pi_{3}\left(X_{1}\right)$ and attach an 8-cell by a map representing the element $N \iota_{c}-\alpha_{1}\left[\left[\iota_{b}, \iota_{a}\right], \iota_{a}\right]-\alpha_{2}\left[\left[\iota_{b}, \iota_{a}\right], \iota_{b}\right]$ in $\pi_{7}\left(X_{1}\right)$.

3. Properties of $T^{*, *}\left(I^{N}\right)$. Denote by $I^{N}$ the standard $N$-cube in $R^{N} . I^{N}$ is the set of $N$-tuples $x=\left(x_{1}, \ldots, x_{N}\right)$ of real numbers $0 \leq x_{i} \leq 1$, $i=1,2, \ldots, N$. A basic $p$-form of weight $q$ on $I^{N}$ in the coordinates 
$x_{1}, \ldots, x_{N}$ is a differential form

$$
x_{i_{1}}^{\alpha_{1}} \cdots x_{i_{j}}^{\alpha_{j}} x_{k_{1}}^{\beta_{1}} d x_{k_{1}} \wedge \cdots \wedge x_{k_{p}}^{\beta_{p}} d x_{k_{p}}
$$

where $\left\{i_{1}, \ldots, i_{j}\right\}$ and $\left\{k_{1}, \ldots, k_{p}\right\}$ are disjoint subsets of $\{1,2, \ldots, N\}$, the $\alpha$ 's and $\beta$ 's are nonnegative integers, and $q=\max \left\{\alpha_{1}, \ldots, \alpha_{j} ; \beta_{1}+\right.$ $\left.1, \ldots, \beta_{p}+1\right\}$.

Denote by $Q_{q}$ the smallest subring of the rationals containing $1 / p$ for each prime $p$ with $p \leq q$. Denote by $T^{p, q}\left(I^{N}\right)$ the module of all $Q_{q}$-linear combinations of basic $p$-forms of weight less than or equal to $q$.

The usual differential on forms extends to a $Q_{q}$-linear operator $d$ : $T^{p, q}\left(I^{N}\right) \rightarrow T^{p+1, q}\left(I^{N}\right)$ and the wedge product operation on basic forms extends to a map

$$
T^{p_{1}, q_{1}}\left(I^{N}\right) \otimes T^{p_{2}, q_{2}}\left(I^{N}\right) \stackrel{\wedge}{\rightarrow} T^{p_{1}+p_{2}, q_{1}+q_{2}}\left(I^{N}\right)
$$

Note that the wedge product of forms of weights $q_{1}$ and $q_{2}$ can have weight less than $q_{1}+q_{2}$. For example the wedge product of form $x_{1}^{2} d x_{2}$ of weight 2 in $I^{2}$ and of form $x_{2}$ of weight 1 in $I^{2}$ is the form $x_{1}^{2} x_{2} d x_{2}$ which has weight 2 . The restriction of a form of weight $q$ on $I^{N}$ to a face of $I^{N}$ can have weight less than $q$. For example the form $x_{2}^{2} d x_{1}$ on $I^{2}$ of weight 2 restricted to the face $x_{2}=1$ is the form $d x_{1}$ on $I^{1}$ which has weight 1 .

Denote the boundary of $I^{N}$ by $\partial I^{N}$ and let $r$ denote the restriction of $(p, q)$ forms on $I^{N}$ to the boundary. The following lemma is used to prove Theorem 4.1.

LEMMA 3.1. For $p \geq 0, q \geq 1$ the sequence $T^{p, q}\left(I^{N}\right) \stackrel{r}{\rightarrow} T^{p, q}\left(\partial I^{N}\right) \rightarrow 0$ is exact. That is, every p-form of weight less than or equal to $q$ on $\partial I^{N}$ is the restriction of a p-form of weight less than or equal to $q$ on $I^{N}$.

The proof of Lemma 3.1 follows easily from

Proposition 3.2. Let $\left(I^{N}\right)^{k}, k \geq 0$, denote the $k$-skeleton of $I^{N}$. Suppose $\omega \in T^{p, q}\left(\left(I^{N}\right)^{k}\right), q \geq 1$, and the restriction of $\omega$ to the $(k-1)$ skeleton is identically zero. Then there is an $\omega_{k}$ in $T^{p, q}\left(I^{N}\right)$ whose restriction to $\left(I^{N}\right)^{k}$ is $\omega$. 
Proof of Lemma 3.1. Assume by induction on $k$ that $T^{p, q}\left(I^{N}\right) \rightarrow$ $T^{p, q}\left(\left(I^{N}\right)^{k-1}\right) \rightarrow 0$ is exact and consider the diagram

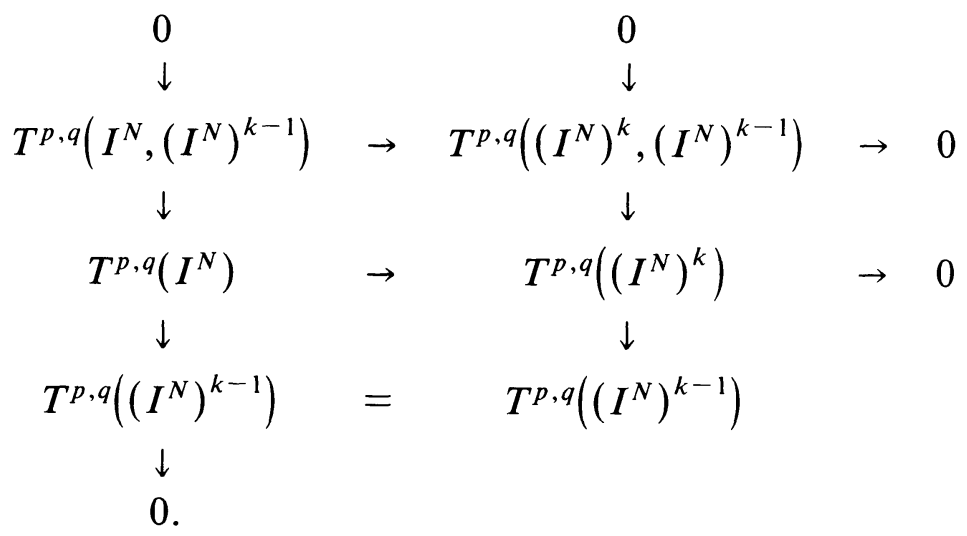

The column on the left is exact by assumption, the top row is exact by Proposition 3.2, and the second column is exact by definition of $T^{p, q}\left(\left(I^{N}\right)^{k},\left(I^{N}\right)^{k-1}\right)$. A diagram chase shows that the middle row is exact. Thus Proposition 3.2 implies $T^{p, q}\left(I^{N}\right) \rightarrow T^{p, q}\left(\left(I^{N}\right)^{k}\right) \rightarrow 0$ is exact for all $k$. In particular, $T^{p, q}\left(I^{N}\right) \rightarrow T^{p, q}\left(\left(I^{N}\right)^{N-1}=\partial I^{N}\right) \rightarrow 0$ is exact.

Proof of Proposition 3.2. Let $\omega \in T^{p, q}\left(\left(I^{N}\right)^{k},\left(I^{N}\right)^{k-1}\right)$ with $q \geq 1$. We want to show that $\omega$ is the restriction of a form $\omega_{k}$ on $I^{N}$. Since $\omega$ restricted to $\left(I^{N}\right)^{k-1}$ is identically zero, $\omega$ is a sum of elements in $T^{p, q}\left(\left(I^{N}\right)^{k},\left(I^{N}\right)^{k-1}\right)$ which are nonzero on only one $k$-face of $\left(I^{N}\right)^{k}$. Thus we can assume $\omega$ is nonzero on only one $k$-face of $I^{N}$, say $F$. Denote by $\left\{x_{a_{1}}, \ldots, x_{a_{k}}\right\}$ the set of variable coordinates on $F$. Set $\left\{x_{b_{1}}, \ldots, x_{b_{l}}\right\}$ equal to the set of coordinates that are identically 1 on $F$ and set $\left\{x_{c_{1}}, \ldots, x_{c_{j}}\right\}$ equal to the set of coordinates that are identically zero on $F$. Note $\left\{a_{1}, \ldots, a_{k}\right\},\left\{b_{1}, \ldots, b_{i}\right\},\left\{c_{1}, \ldots, c_{j}\right\}$ are disjoint sets whose union is $\{1,2,3, \ldots, N\}$. $\omega$ restricted to $F$ is a sum of forms of type $p\left(x_{a_{1}}, \ldots, x_{a_{k}}\right) d x_{a_{l_{1}}} \wedge \cdots \wedge d x_{a_{t_{p}}}$, where $p\left(x_{a_{1}}, \ldots, x_{a_{k}}\right)$ is a polynomial with $Q_{q}$ coefficients and $1 \leq i_{1}{ }^{p}<i_{2}<\cdots<i_{p} \leq k$. Since $\omega$ restricted to any $(k-1)$-face of $F$ is zero, it follows that each one of the summands restricted to a $(k-1)$-face is also zero. Thus it is sufficient to prove Proposition 3.2 in the special case where $\omega=p\left(x_{a_{1}}, \ldots, x_{a_{k}}\right) d x_{a_{1}}$ $\wedge \cdots \wedge d x_{a_{p}}$, where $\omega$ is nonzero only on the $k$-face $F$ of $I^{N}$, and where $\omega$ vanishes on the $(k-1)$-skeleton of $F$. The condition that $\omega$ vanishes on the $(k-1)$-skeleton implies that if $x_{a_{i}}$ is 1 or 0 with $p+1 \leq i \leq k$, then $p\left(x_{a_{1}}, \ldots, x_{a_{p}}, \ldots, x_{a_{t}}, \ldots, x_{a_{k}}\right)=0$. 
Define the element $\omega_{k} \in T^{p, q}\left(I^{N}\right)$ by the formula

$$
\omega_{k}=x_{b_{1}} \cdots x_{b_{i}}\left(1-x_{c_{1}}\right) \cdots\left(1-x_{c_{J}}\right) p\left(x_{a_{1}}, \ldots, x_{a_{k}}\right) d x_{a_{1}} \wedge \cdots \wedge d x_{a_{p}} .
$$

Clearly $\omega_{k}$ restricted to $F$ equals $\omega$. We claim that the restriction of $\omega_{k}$ to any other $k$-face of $I^{N}$, say $F^{1}$, is identically zero. If $\left\{x_{b_{1}} \cdots x_{b_{t}}, x_{c_{1}}, \ldots, x_{c_{l}}\right\}$ are the variables that are constant on $F^{1}$, then $x_{b_{1}}, \ldots, x_{b_{l}}\left(1-x_{c_{k}}\right) \cdots$ $\left(1-x_{c_{j}}\right) \equiv 0$ on $F^{1}$ so $\omega_{k}$ restricted to $F^{1}$ is identically zero. Suppose $\left\{x_{b_{1}}, \ldots, x_{b_{i}}, x_{c_{1}}, \ldots, x_{c}\right\}$ is not the set of constant variables on $F^{1}$. Then at least one of the coordinate functions $\left\{x_{a_{1}}, \ldots, x_{a_{k}}\right\}$ must be constant on $F^{1}$. If $x_{a_{i}}$ is constant, $1 \leq i \leq p$, then $d x_{a_{1}} \wedge \cdots \wedge d x_{a_{l}} \wedge \cdots \wedge d x_{a_{p}}=0$ on $F^{1}$ so $\omega_{k}=0$ on $F^{1}$. If $x_{a_{i}}$ is constant, $p+1 \leq i \leq k$, then $p\left(x_{a_{1}}, \ldots, x_{a_{k}}\right)=0$ on $F^{1}$ since $\omega$ vanishes on the $(k-1)$-skeleton.

If $J$ is any $(k-1)$-face of $I^{N}$ then at least one of the variables $x_{a_{t}}$ must be constant on $J$. But then $\omega_{k}$ restricted to $J$ is identically zero by the above argument.

Proposition 3.3. Let $\omega$ be $a(p, q)$-form on $I^{N}$. Then the integral of $\omega$ over any p-face of $I^{N}$ is an element of the ring $Q_{q}$.

Proof. We can restrict ourselves to the $(p, q)$-form

$$
\omega=x_{i_{1}}^{\alpha_{1}} \cdots x_{l_{j}}^{\alpha_{j}} x_{k_{1}}^{\beta_{1}} d x_{k_{1}} \wedge \cdots \wedge x_{k_{p}}^{\beta_{p}} d x_{k_{p}} .
$$

A $p$-face $P$ of $I^{N}$ is determined by specifying $p$ variable coordinates and from the remaining $N-p$ coordinates specifying some to be equal to 1 and the rest to be equal to 0 . If the $p$ variable coordinates are not $x_{k_{1}}, \ldots, x_{k_{p}}$, then the integral of $\omega$ over $P$ is equal to zero. If the coordinates $x_{k_{1}}, \ldots, x_{k_{p}}$ are variable, then the restriction of $\omega$ to $P$ is either zero or equal to

$$
\omega^{1}=x_{k_{1}}^{\beta_{1}} d x_{k_{1}} \wedge \cdots \wedge x_{k_{p}}^{\beta_{p}} d x_{k_{p}} .
$$

If $P$ is oriented by the ordered basis $\left\{d x_{k_{1}}, \ldots, d x_{k_{p}}\right\}$, then

$$
\begin{aligned}
\int_{P} \omega^{1} & =\left(\int_{0}^{1} x_{k_{1}}^{\beta_{1}} d x_{k_{1}}\right)\left(\int_{0}^{1} x_{k_{2}}^{\beta_{2}} d x_{k_{2}}\right) \cdots\left(\int_{0}^{1} x_{k_{p}}^{\beta_{p}} d x_{k_{p}}\right) \\
& =\left(\beta_{1}+1\right)^{-1}\left(\beta_{2}+1\right)^{-1} \cdots\left(\beta_{p}+1\right)^{-1} .
\end{aligned}
$$

This is an element of $Q_{q}$ since each $\beta_{i}+1 \leq q$. 
Because the proof of Stokes' Theorem for chains on $I^{N}$ can be repeated word for word from the classical case we get

COROLlaRY 3.4. The integration over chains induces a map of cochain complexes

$$
I: T^{*, q}\left(I^{N}\right) \rightarrow C^{*}\left(I^{N} ; Q_{q}\right),
$$

where on the right we have the complex of cubical cochains on $I^{N}$.

Proposition 3.5. For $N \geq 1$ and $q \geq 1$, the cohomology of the complex $\left\{T^{*, q}\left(I^{N}\right), d\right\}$ is given by

$$
H^{\prime}\left(T^{*, q}\left(I^{N}\right)\right)= \begin{cases}Q_{q} & \text { for } i=0, \\ 0 & \text { for } i>0 .\end{cases}
$$

Proof. The result follows by direct calculation in the case $N=1$. Assume by induction that the proposition holds for $N=k$. The proposition follows for $N=k+1$ by applying the Kunneth Theorem to $T^{*, q}\left(I^{k}\right)$ $\otimes_{Q_{q}} T^{*, q}\left(I^{1}\right)=T^{*, q}\left(I^{k+1}\right)$.

4. Properties of $T^{*, *}(X)$. Let $X$ be a simplicial complex of finite type together with a subdivision into nondegenerate cubes. An element in $T^{p, q}(X)$ is a rule, $w$, which assigns to each nondegenerate $N$-cube, $F$, in $X$ an element $\omega(F)$ in $T^{p, q}\left(I^{N}\right)$ so that for each face $J$ of $F, \omega(J)$ is the restriction of $\omega(F)$ to the face $J . \omega$ is called a $(p, q)$ form on $X$. Integration gives a map of the complex $T^{*, q}(X)$, of forms of weight $\leq q$ on $X$, into the complex of cubical cochains $C^{*}\left(X ; Q_{q}\right)$ on $X$ with $Q_{q}$ coefficients:

$$
I: T^{*, q}(X) \rightarrow C^{*}\left(X ; Q_{q}\right) .
$$

From Corollary 3.4 it follows that this is a map of cochain complexes. In fact in this case we have

THEOREM 4.1. For $q \geq 1$ the map

$$
I: H^{p}\left(T^{* q,}(X)\right) \rightarrow H^{p}\left(X ; Q_{q}\right)
$$

induced by integration is an isomorphism for all $p$.

Proof. The proof is by induction on the skeletons of $X$. Suppose the proposition is true on the $l$-skeletons, $X^{l}$, of $X$ for $l<k$. 
Consider the commutative diagram

$$
\begin{array}{ccccccccc}
0 & \rightarrow & T^{*, q}\left(X^{k}, X^{k-1}\right) & \rightarrow & T^{*, q}\left(X^{k}\right) & \rightarrow & T^{*, q}\left(X^{k-1}\right) & \rightarrow & 0 \\
& & I \downarrow & & I \downarrow & & I \downarrow & & \\
0 & \rightarrow & C^{*}\left(X^{k}, X^{k-1} ; Q_{q}\right) & \rightarrow & C^{*}\left(X^{k} ; Q_{q}\right) & \rightarrow & C^{*}\left(X^{k-1} ; Q_{q}\right) & \rightarrow & 0
\end{array}
$$

which gives rise to the commutative diagram

$$
\begin{aligned}
& \cdots \rightarrow H^{p-1}\left(T^{* \cdot q}\left(X^{k-1}\right)\right) \stackrel{\delta}{\rightarrow} H^{p}\left(T^{*} \cdot q\left(X^{k}, X^{k-1}\right)\right) \rightarrow H^{p}\left(T^{*} \cdot q\left(X^{k}\right)\right) \rightarrow H^{p}\left(T^{*} \cdot q\left(X^{k-1}\right)\right) \rightarrow \cdots
\end{aligned}
$$

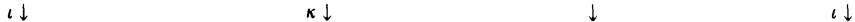

$$
\begin{aligned}
& \ldots \rightarrow H^{p-1}\left(X^{k-1} ; Q_{q}\right) \stackrel{\delta}{\rightarrow} \quad H^{p}\left(X^{k}, X^{k-1} ; Q_{q}\right) \quad \rightarrow \quad H^{p}\left(X^{q} ; Q_{q}\right) \quad \rightarrow \quad H^{p}\left(X^{k-1} ; Q_{q}\right) \rightarrow \cdots
\end{aligned}
$$

where the rows are exact and $\iota$ is an isomorphism by assumption. By the five lemma it suffices to show that $\kappa$ is an isomorphism. Let $\left\{I_{j}^{k} ; j \in J\right\}$ be the set of $k$-cubes of $X^{k}$. Then as a $Q_{q}$-module

$$
T^{*, q}\left(X^{k}, X^{k-1}\right)=\sum_{j} T^{*, q}\left(I_{j}^{k}, \partial I_{j}^{k}\right)
$$

and

$$
C^{*}\left(X^{k}, X^{k-1} ; Q_{q}\right)=\sum_{J} C^{*}\left(I_{j}^{k}, \partial I_{j}^{k} ; Q_{q}\right)
$$

and it is sufficient to show that integration induces an isomorphism $H^{p}\left(T^{*, q}\left(I^{k}, \partial I^{k}\right)\right) \stackrel{\tau}{\rightarrow} H^{p}\left(I^{k}, \partial I^{k} ; Q_{q}\right)$.

The commutative diagram

$$
\begin{aligned}
& 0 \rightarrow T^{*, q}\left(I^{k}, \partial I^{k}\right) \quad \rightarrow \quad T^{*, q}\left(I^{k}\right) \quad \rightarrow \quad T^{*, q}\left(\partial I^{k}\right) \quad \rightarrow \quad 0 \\
& I \downarrow \quad I \downarrow \quad I \downarrow \\
& 0 \rightarrow C^{*}\left(I^{k}, \partial I^{k} ; Q_{q}\right) \rightarrow C^{*}\left(I^{k} ; Q_{q}\right) \rightarrow C^{*}\left(\partial I^{k} ; Q_{k}\right) \rightarrow 0
\end{aligned}
$$

gives rise to a map of long exact cohomology sequences (note the top row is exact by Lemma 3.1) with $I: H^{*}\left(T^{*, q}\left(I^{k}\right)\right) \rightarrow H^{*}\left(I^{k} ; Q_{q}\right)$ an isomorphism by Proposition 3.5. Hence $H^{*}\left(T^{*, q}\left(I^{k}, \partial I^{k}\right)\right) \stackrel{\tau}{\rightarrow} H^{*}\left(I^{k}, \partial I^{k} ; Q_{q}\right)$ is an isomorphism by the five lemma.

If we denote by $\wedge$ the product on cohomology induced by the wedge product of differential forms and by $U$ the cup product, then we can 
formulate the multiplicativity property of the isomorphism $I$ as follows:

THEOREM 4.2. The diagram

$$
\begin{array}{ccc}
H^{\prime}\left(T^{*, q_{1}}(X)\right) \otimes H^{j}\left(T^{*, q_{2}}(X)\right) & \stackrel{\wedge}{ } & H^{i+j}\left(T^{*, q_{1}+q_{2}}(X)\right) \\
I \otimes I \downarrow & & I \downarrow \\
H^{i}\left(X ; Q_{q_{1}}\right) \otimes H^{J}\left(X ; Q_{q_{2}}\right) & \stackrel{\cup}{ } & H^{i+j}\left(X ; Q_{q_{1}+q_{2}}\right)
\end{array}
$$

commutes.

Proof. It is sufficient to show that the diagram

$$
\begin{array}{ccc}
H^{p_{1}}\left(X: Q_{q_{1}}\right) \otimes H^{p_{2}}\left(X: Q_{q_{2}}\right) & \stackrel{\cup}{ } & H^{p_{1}+p_{2}}\left(X: Q_{q_{1}+q_{2}}\right) \\
J \otimes J \downarrow & & \uparrow I \\
H^{p_{1}}\left(T^{*, q_{1}}(X)\right) \otimes H^{p_{2}}\left(T^{*, q_{2}}(X)\right) & \stackrel{\leftrightarrow}{\rightarrow} & H^{p_{1}+p_{2}}\left(T^{*, q_{1}+q_{2}}(X)\right)
\end{array}
$$

commutes, where $J$ denotes the inverse of $I . J$ is induced by a map $J$ : $C^{*}\left(X ; Q_{q}\right) \rightarrow T^{*, q}(X)$ of cochain complexes. For $i=\left(i_{1}, i_{2}, \ldots, i_{N-p}\right)$ a sequence of integers with $1 \leq i_{1}<i_{2}<\cdots<i_{N-p} \leq N$ and $\varepsilon=$ $\left(\varepsilon_{1}, \varepsilon_{2}, \ldots, \varepsilon_{N-p}\right)$ a sequence of 0 's and l's, denote by $\lambda_{i}^{\varepsilon}$ the $p$-face of $I^{N}$ with the $i_{j}$ th coordinate function, $X_{i}$, identically equal to $\varepsilon_{j}$ for $1 \leq j \leq N$ - $p$. Denote by $\left(\lambda_{i}^{\varepsilon}\right)^{*}$ the element of $C^{p}\left(I^{N} ; Z\right)$ which is 1 on $\lambda_{i}^{\varepsilon}$ and 0 on all other $p$-faces on $I^{N}$. Set $J\left(\lambda_{i}^{\varepsilon}\right)^{*}$ equal to

$$
y_{1} y_{2} \cdots y_{N-p} d x_{j_{p}} \cdots d x_{j_{p}} \quad \text { where } y_{j}= \begin{cases}x_{i_{j}} & \text { if } \varepsilon_{j}=1, \\ 1-x_{i_{j}} & \text { if } \varepsilon_{j}=0\end{cases}
$$

and $\left(j_{1}, j_{2}, \ldots, j_{p}\right)$ is the increasing sequence with $\left\{i_{1}, \ldots, i_{N-p}\right\} \cup$ $\left\{j_{1}, \ldots, j_{p}\right\}=\{1,2, \ldots, N\}$. For $q \geq 1$ the map $J: C^{*}\left(I^{N} ; Q_{q}\right) \rightarrow T^{*, q}\left(I^{N}\right)$ of cochain complexes extends uniquely to a map $J: C^{*}\left(X ; Q_{q}\right) \rightarrow T^{*, q}(X)$ of cochain complexes. For $F$ a $p$-face of $I^{N}$,

$$
\int_{F} J\left(\lambda_{i}^{\varepsilon}\right)^{*}= \begin{cases}1 & \text { if } F=\lambda_{i}^{\varepsilon} \\ 0 & \text { otherwise }\end{cases}
$$

Hence $I \circ J$ is the identity on $C^{*}\left(X ; Q_{q}\right)$.

Define a map $\phi: C_{*}(X ; Z) \rightarrow Q_{2} \otimes C_{*}(X ; Z) \otimes C_{*}(X ; Z)$ as follows. 
Let $N$ be an integer, $N>0$. Let $i=\left(i_{1}, \ldots, i_{p}\right), j=\left(j_{1}, \ldots, j_{N-p}\right)$ be increasing sequences of integers from the set $\{1,2, \ldots, N\}$. Let $\varepsilon=$ $\left(\varepsilon_{1}, \ldots, \varepsilon_{p}\right), \varepsilon^{1}=\left(\varepsilon_{1}^{1}, \ldots, \varepsilon_{N-p}^{1}\right)$ be sequences of 0 's and 1's. Define the element $a\left(i, \varepsilon, j, \varepsilon^{1}\right)$ of $Q_{2}$ by

$$
a\left(i, \varepsilon, j, \varepsilon^{1}\right)= \begin{cases}0 & \text { if } i \cap j \neq \varnothing \\ \frac{1}{2^{N}} & \text { if } i \text { or } j \text { is empty } \\ \frac{(-1)^{\rho(i, j)}}{2^{N}} & \text { otherwise }\end{cases}
$$

where $\rho(i, j)$ is the number of pairs $\left(j_{k}, i_{l}\right)$ with $i_{l}<j_{k}$. Define

$$
\phi: C_{*}\left(I^{N} ; Z\right) \rightarrow Q_{2} \otimes C_{*}\left(I^{N} ; Z\right) \otimes C_{*}\left(I^{N} ; Z\right)
$$

by setting

$$
\phi\left(I^{N}\right)=\sum a\left(i, \varepsilon, j, \varepsilon^{1}\right) \otimes \lambda_{i}^{\varepsilon} \otimes \lambda_{j}^{\varepsilon^{1}}
$$

where the sum is over all pairs $\left(\lambda_{i}^{\varepsilon}, \lambda_{j}^{\varepsilon^{1}}\right)$ of faces of $I^{N}$ whose dimensions add up to $N$, and by requiring that $\phi$ commute with the inclusion of faces of $I^{N}$ into $I^{N}$. This defines $\phi: C_{*}(X ; Z) \rightarrow Q_{2} \otimes C_{*}(X ; Z) \otimes C_{*}(X ; Z)$. $\phi$ induces a map $C^{*}(X ; Z) \otimes C^{*}(X ; Z) \rightarrow C^{*}\left(X ; Q_{2}\right)$. From the acyclic models theorem [8] it follows that any two diagonal approximations induce the same product on cohomology. Hence the map $H^{*}(X ; Z) \otimes$ $H^{*}(X ; Z) \stackrel{\phi^{*}}{\rightarrow} H^{*}\left(X ; Q_{2}\right)$ is the standard cup product [13] followed by the coefficient map induced by the inclusion of $Z$ into $Q_{2}$.

By a direct computation

$$
a\left(i, \varepsilon, j, \varepsilon^{1}\right)=\int_{I^{N}} J\left(\lambda_{i}^{\varepsilon}\right)^{*} \wedge J\left(\lambda_{J}^{\varepsilon^{1}}\right) * .
$$

Hence diagram (1) is commutative on the level of cochain complexes.

ReMark. The map $\phi: C_{*}\left(X ; Q_{2}\right) \rightarrow C_{*}\left(X ; Q_{2}\right) \otimes C_{*}\left(X ; Q_{2}\right)$ is not co-associative. The map of chain complexes

$$
E: C_{p}\left(X ; Q_{q}\right) \rightarrow \operatorname{Hom}_{Q_{q}}\left(T^{p, q}(X) ; Q_{q}\right)
$$

is defined as follows: Let $\sigma=\sum \sigma_{l} \otimes a_{l}$ be an element of $C_{p}(X) \otimes Q_{q}(X$ of finite type). Set $E(\sigma)$ equal to the homomorphism which, when applied to an element $\omega$ in $T^{p, q}(X)$, is the element $\Sigma a_{l} \int_{\sigma_{l}} \omega$ of $Q_{q}$. It follows from Corollary 3.4 that $E$ is a map of chain complexes. 
THEOREM 4.3. If $X$ is a simplicial complex of finite type, the map $E: H_{q}\left(X ; Q_{q}\right) \rightarrow H_{p}\left(T^{*, q}(X)\right)$ is an isomorphism of $Q_{q}$-modules for $q \geq 1$ and all $p$.

Proof. From the universal coefficient theorem for cohomology we get the commutative diagram with exact rows:

$$
\begin{aligned}
& 0 \rightarrow \operatorname{Ext}_{Q_{q}}\left(H^{p+1}\left(T^{* . q}(X)\right), Q_{q}\right) \rightarrow H_{p}\left(T^{* . q}(X)\right) \rightarrow \operatorname{Hom}_{Q_{q}}\left(H^{p}\left(T^{* . q}(X)\right) ; Q_{q}\right) \rightarrow 0 \\
& 0 \rightarrow \operatorname{Ext}_{Q_{q}}\left(H^{p+1}\left(X ; Q_{q}\right), Q_{q}\right) \rightarrow H_{p}\left(X ; Q_{q}\right) \rightarrow \operatorname{Hom}_{Q_{q}}\left(H^{p}\left(X ; Q_{q}\right) ; Q_{q}\right) \rightarrow 0
\end{aligned}
$$

where $i_{\mathrm{Ext}}$ and $i_{H}$ are isomorphisms. Hence $E$ is an isomorphism.

\section{REFERENCES}

[1] H. Cartan, Théorie cohomologiques, Inventiones Math., 35 (1976), 261-271.

[2] B. Cenkl and R. Porter, Complétion de Lazard des groupes par des formes differentielles, C.R. Acad. Sc. Paris, 288 (1979), Serie A, 445-447.

[3] _ Differential forms and torsion in the fundamental group, Advances in Math., 48 (1983), 189-204.

[4] _ Modèles pour la théorie de l'homotopie moderée, C.R. Acad. Sc. Paris, 290 (1980), Série A, 613-615.

[5] _ Tame homotopy; Colecao Atas (Sociedade Brasileira De Mathemática Segundo Encontro Brasileiro De Topologia), 13 (1980), 1-32.

[6] __ Algebraic categories and homotopy theory, to appear in the proceedings of the conference on homotopy theory held in Luminy, June 1982.

[7] W. G. Dwyer, Tame homotopy theory, Topology, 18 (1979), 321-338.

[8] S. Eilenberg and S. MacLane, Acyclic Models, Amer. J. Math., 75 (1953), 189-199.

[9] S. Halperin and J. Stasheff, Obstructions to homotopy equivalence, Advances in Math., 32 (1979), 233-279.

[10] L. Lambe and S. Priddy, Cohomology of nilmanifolds and torsion-free, nilpotent groups, (preprint 1982).

[11] E. Y. Miller, deRham cohomology with arbitrary coefficients, Topology, 17 (1978), 193-203.

[12] M. Schlessinger and J. Stasheff, Deformation theory and rational homotopy type, I.H.E.S. (preprint).

[13] J. P. Serre, Homologie singuliere des espaces fibres, applications, Annals of Math., 54 (1951), 425-505.

[14] D. Sullivan, Infinitesimal computations in topology, Pub. Math. I.H.E.S., 47 (1978), 269-332.

Received March 13, 1979 and in revised form February 16, 1983. The first author was supported in part by the National Science Foundation under NSF-MCS77-04945.

\section{NORTHEASTERN UNIVERSITY}

BosTon, MA 02115 


\section{PACIFIC JOURNAL OF MATHEMATICS \\ EDITORS}

DONALD BABBITT (Managing Editor)

University of California.

Los Angeles, CA 90024

Hugo Rossi

University of Utah

Salt Lake City, UT 84112

C. C. Moore and Arthur Ogus

University of California

Berkeley, CA 94720
J. DugundjI

Department of Mathematics

University of Southern California

Los Angeles, CA 90089-1113

R. FinN and H. Samelson

Stanford University

Stanford, CA 94305

\section{ASSOCIATE EDITORS}
R. ARENS
E. F. BECKENBACH
B. H. NeumanN
F. WOLF
K. YoshidA (1906-1982)

\section{SUPPORTING INSTITUTIONS}

UNIVERSITY OF ARIZONA

UNIVERSITY OF BRITISH COLUMBIA

CALIFORNIA INSTITUTE OF TECHNOLOGY

UNIVERSITY OF CALIFORNIA

MONTANA STATE UNIVERSITY

UNIVERSITY OF NEVADA. RENO

NEW MEXICO STATE UNIVERSITY

OREGON STATE UNIVERSITY
UNIVERSITY OF OREGON

UNIVERSITY OF SOUTHERN CALIFORNIA

STANFORD UNIVERSITY

UNIVERSITY OF HAWAII

UNIVERSITY OF TOKYO

UNIVERSITY OF UTAH

WASHINGTON STATE UNIVERSITY

UNIVERSITY OF WASHINGTON 


\section{Pacific Journal of Mathematics}

Vol. 112, No. $1 \quad$ January, 1984

Richard Blaine Barrar and Henry Loeb, Characterizing the divided difference weights for extended complete Tchebycheff systems $\ldots \ldots \ldots \ldots 1$

Harold Bennett and David John Lutzer, Generalized ordered spaces with

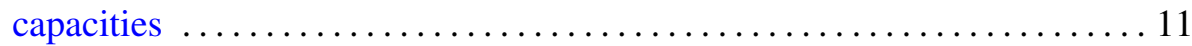

Geoffrey J. Butler and Lynn Harry Erbe, Comparison theorems for second-order operator-valued linear differential equations

Bohumil Cenkl and Richard D. Porter, de Rham theorem with cubical

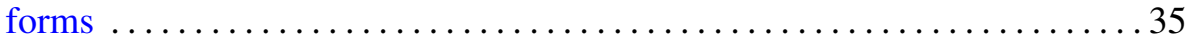

Zvonko Cerin, Characterizing global properties in inverse limits . ....... 49

Thomas Eugene Duchamp and Morris Kalka, Holomorphic foliations and deformations of the Hopf foliation .........................69 69

John Paul Hempel, Homology of coverings $\ldots \ldots \ldots \ldots \ldots \ldots \ldots \ldots \ldots$

Gerald Norman Hile and R. Z. Yeh, Inequalities for eigenvalues of the

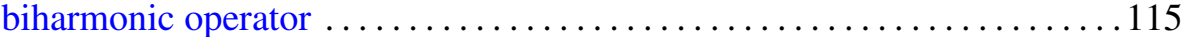

Kenneth Irwin Joy, A description of the topology on the dual space of a

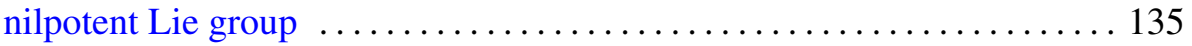

Alex Kumjian, On localizations and simple $C^{*}$-algebras $\ldots \ldots \ldots \ldots \ldots 141$

Bernardus de Pagter, The space of extended orthomorphisms in a Riesz

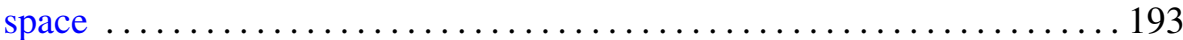

Stephen C. Persek, Iterated averaging for periodic systems with hidden

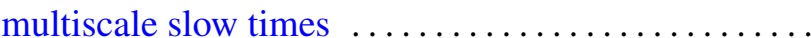

David Francis Rearick, Divisibility of arithmetic functions . . . . . . . . . 237

Masaaki Suzuki, The intrinsic metrics on the circular domains in $\mathbf{C}^{n}$ 